\title{
Was passieren muss
}

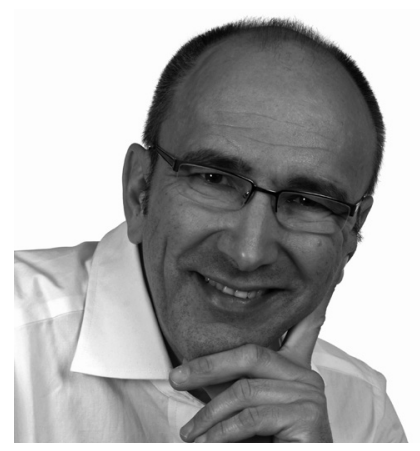

VON STEFAN BLANK

Stefan Blank ist Geschäftsführer des "PsychoSozialen Netzwerks" in Ludwigsburg. Seine Arbeitsschwerpunkte liegen im strategischen Management und in der Umsetzung personenzentriert und sozialräumlich wirksamer Versorgungskulturen in der Sozialpsychiatrie.

info@reifezeit.eu

\author{
Inklusion, Sozialraum und Personenorientierung \\ scheinen die neuen Leitideen von Gesellschaft, \\ Politik und Sozialwirtschaft zu sein. Die Stolpersteine \\ auf dem Weg zu diesen Zielen hat der Autor in \\ der letzten Ausgabe in zwölf Thesen beschrieben. \\ In diesem Beitrag skizziert er in weiteren zwölf \\ Thesen, was aus Sicht eines Verantwortlichen \\ eines Sozialunternehmens passieren sollte.
}

\begin{abstract}
»Hoffnung ist nicht die Überzeugung, dass etwas gut ausgeht, sondern die Gewissheit, dass etwas Sinn hat, egal wie es ausgeht.« - Vaclav Havel
\end{abstract}

1 Mietrecht hat immer Vorrang vor 1. Heimrecht. Ein selbstbestimmtes Wohnrecht an allen Orten ist der Vorrang vor dem Bezug von Leistungen in fremdbestimmten Sonderwelten einzuräumen. Wir brauchen dafür einen sozialen regionalen Wohnungsbau mit Belegrechten in Hilfeplankonferenzen anstatt eines wohlfahrtsverbandlichen Wohnheimbaus.

2 Zeitbasierte - und damit teilbare 2. Leistungen haben Vorrang vor platzzahlorientierten unteilbaren Pauschalleistungen. Persönliche Budgets und Regionalbudgets als gesetzliche Vorgaben verändern die Planungsvorhaben und die Zahlungsströme - und verhindern Sonderwelten.

3. Soziale Kontakträume an allen Orten, Beschäftigungs-und Arbeitsräume lassen sich durch einen Investitionskostenzuschlag je Leistungsstunde "mobilisieren «, also überall dort, wo die behinderten Menschen wohnen oder arbeiten wollen, lassen sich Werkstätten und Begegnungsstätten anmieten. Einschränkend ist bei psychisch behinderten Menschen darauf zu achten, dass mit einer einsetzenden Stabilisierung, der Indi- vidualanspruch auf Eingliederungshilfe »individuell abnimmt « oder ganz endet. Dann braucht es »Grundleistungen «, Tages- und Kontaktstätten, in denen sich behinderte Menschen (erfreulicherweise) ohne Leistungsbezug treffen können.

1 Eine einheitliche Leistungssyste4. matik ist zeitbasiert neu auszurichten. Die Bedarfsbemessung muss intensivere Hilfe als in einer stationären Einrichtung und eine nachsorgende "ausschleichende " Hilfe als in pauschalen ambulanten Vergütungssystemen ermöglichen. Das dezeitige Hilfesystem kennt - beim Vergleich mit einem Automatik-Siebenganggetriebe beim Auto - nur den zweiten Gang und den siebten Gang. Die anderen Gänge (also Leistungsstufen) sind blockiert.

5. Ein uneingeschränkter bürger5. licher Sozialrechtsstatus (z. B. Recht auf eine Mietwohnung) hat stets Vorrang vor leistungs- und menschenrechtlichen Einschränkungen der stationären Eingliederungshilfe. Wir brauchen eine selbstbestimmte - gleichfalls sicher zu organisierende - unterstützte Versorgung statt einrichtungszentrierter All-inclusive-Versorgung.

6 Leistungen der Eingliederungshil6. fe und Teilhabe müssen auch für Menschen gelten, die die aus Kosten- 
gründen in Pflegeeinrichtungen verlegt werden. Individuelle sozialraum- und personenzentrierte Teilhabe ist zu organisieren; sie hat allemal Vorrang vor einrichtungszentrierten statischen Versorgungsstrukturen.

7. Mieter-sein-Dürfen bedeutet - Gleichberechtigt-sein-Können im Sinne der UN-Konvention. Das Wunschund Wahlrecht, die Bedürfnisse von Menschen mit Behinderungen haben Vorrang vor fürsorgeorientierten Angeboten der Kostenträger oder Leistungserbringer (Teilhabeplanung). Neutrale Beratungsleistungen zur Hilfeplanung haben Vorrang vor Beratungen durch Einrichtungen oder Kostenträger, die immer - berechtigte - Eigeninteressen verfolgen. Hilfeplankonferenzen haben Vorrang vor freihändiger Hilfeplanung einzelner Kostenträger; sind aber erst sinnvoll, wenn die zeitbasierte Vergütung verwirklicht ist (These 2).

8. Vielfältige, vom Leistungsumfang und der Leistungsart fiskalisch teilbare Leistungen der Sozial- und Eingliederungshilfe sind die Voraussetzung, damit sozialräumliche Entwicklungen entstehen können. Sie haben Vorrang vor unteilbaren Vergütungssätzen in Einrichtungen. Zeit vor Platz: Trägerübergreifende personenzentrierte Leistungsbeteiligungen individuell $\mathrm{zu}$ bemessender Leistungsmengen (Zeit) im Sozialraum haben Vorrang vor ortsbindender, die Selbstbestimmungsmöglichkeiten einschränkender Einrichtungszentrierung (Platz). Personenzentrierte Hilfeplanungsdaten im Einzelfall müssen in der Summe aller Fälle die vorrangige Planungsgrundlage im Sinne der Kundenorientierung sein; vor Einschätzungen zur Bedarfslage, die sich an den Kostenträgern oder Leistungserbringern orientieren. Auf der Grundlage der Kundenwünsche ist die Grundlage für Teilhabeplanung der Kommune zu schaffen.

9. Die Förderung der Entwicklung verbindlicher Kooperationsstrukturen in den Gebietskörperschaften muss jeder materiellen Investitionsförderung eines institutionszentrierten Immobilienausbaus vorausgehen. Wettbewerb herrscht in der Institutionszentrierung. Allein die bürgerliche Gesellschaft kann Teilhabe in Einzelfällen leisten. Mit Hilfe der Eingliederungshilfe müssen
Teilhabeleistungen unter Einbindung und Vergütung fähiger Bürger der Gesellschaft gezielt initiiert werden (etwa 10 bis 20 Prozent des Leistungsvolumens). Die angemessene Vergütung von bürgerschaftlichen Leistungen ist die Grundlage dafür, dass sich Teilhabe auch für die Gesellschaft »lohnt" und die Bereitschaft, sich zu beteiligen dauerhaft anhält.

\section{0} Verlässliche Kooperationsme- chanismen unter den Anbietern im Sozialraum brauchen einen Vorrang vor einrichtungszentrierter Dienst- und Leistungsplanung. Selbstbestimmtes und vor allem bedarfs-und leistungsgerechtes - Arbeiten in der Mitte der Gesellschaft hat Vorrang vor Beschäftigung, die über die Ausgleichsabgabe finanziert wird. Reine Bankfinanzierungen müssen Vorrang haben vor der Zweckbindung durch Mittel aus der Ausgleichsabgabe, da sonst eine gesellschaftliche gemeinsame Nutzung der Ressource ausgeschlossen ist. Wo Einzelfallhilfe zurückgehen muss und stationäre Strukturen vermieden werden sollen, bedarf es der Sicherstellung von Kontakt und Beschäftigungsorten in jedem Sozialraum als Grundleistung der Daseinsfürsorge. Sie müssen den gleichen Verpflichtungscharakter haben wie stationäre Angebote (z. B. Tagesstätten).

11. Die öffentliche Hand, insbeson- dere die Bundesländer, haben drei Aufgaben: das Vertrauen unter Leistungserbringern $\mathrm{zu}$ ermöglichen (Kooperationsstrukturen statt Wettbewerbsstrukturen), eine Versorgungskultur zu entwickeln (Koordinieren von Leistungen statt finanzieller Steuerung von Leistungen), die kurzsichtigen Eigeninteressen abzulegen und sich auf das Gemeinwohl zu besinnen (Infrastruktur entwickeln statt Geld sparen wollen).

12 Eine verantwortliche sozial•räumliche Entwicklung muss die Wohnbaugesellschaften in die Verantwortung nehmen. Der institutionszentrierte Nachhol- und Ausbaubedarf muss an sozialräumliche Standards gebunden werden. Jedem Landkreis sollte die Einstellung eines Sozialraumkoordinators für Behindertenhilfe mit der Verpflichtung angeboten werden, in die Finanzierung mit einzusteigen. Die Anstellung sollte bei einem rechtlich selbstständigen
Trägerverbund aller Versorgungsbeteiligten erfolgen. Dort kann auch die unabhängige Beratungsstelle für Menschen mit Behinderungen unter Beteiligung der Trägerfachkräfte und der Fallmanager der Landkreise in kooperativen Netzwerkstrukturen aufgebaut werden.

\section{Fundamente des Sozialstaats}

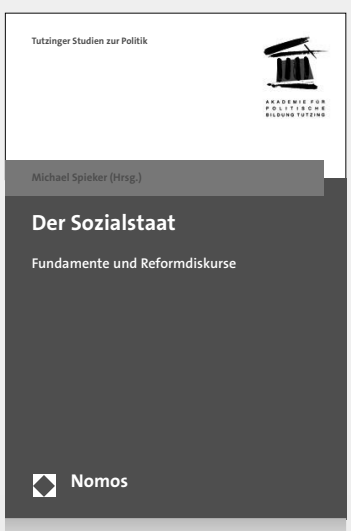

\section{Der Sozialstaat}

Fundamente und Reformdiskurse

Herausgegeben von

Michael Spieker

2012, 305 S., brosch., 49,- $€$

ISBN 978-3-8329-7215-8

(Tutzinger Studien zur Politik, Bd. 4)

Der Einrichtung des Sozialstaats scheint ihre Begründung noch zu fehlen. Im Zuge von „Reformen“ werden die sozialstaatlichen Prinzipien leichtfertig durch unreflektierte Vorstellungen ersetzt. Der Band beleuchtet aus philosophischer, soziologischer, ökonomischer, juristischer und politikwissenschaftlicher Perspektive Begründungen und Herausforderungen des Sozialstaats.

www.nomos-shop.de/14351

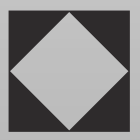

Nomos 